\title{
A self-immolative linker for heparanase activatable probes
}

\author{
Kelton A. Schleyer, "Jun Liu, ZZhishen Wang, and Lina Cui* \\ Department of Medicinal Chemistry, College of Pharmacy, UF Health Science Center, UF Health Cancer \\ Center, University of Florida, Gainesville, FL 32610, USA \\ "These authors contributed equally to the work. \\ *Corresponding Author: Prof. Lina Cui (linacui@cop.ufl.edu)
}

\begin{abstract}
Substrate-based probes utilize known substrate specificity parameters to create a probe that can be activated by a target enzyme. In developing probes for heparanase, an endo-ß-glucuronidase, we previously reported that small, inactive substrate-based probes could be electronically tuned by incorporating electron-withdrawing atoms on the aromatic aglycone fluorophore, ortho- to the cleaved glycosidic bond. However, the installation of electron-withdrawing groups directly onto established fluorophores or other reporters complicates the synthesis of new heparanase probes. In this work we report a new design strategy to expand the toolkit of heparanase imaging probes, in which the installation of an electronically tuned benzyl alcohol linker restored the activity of a previously inactive heparanase probe using 4-methylumbelliferone as the fluorescent reporter, suggesting such a linker can provide a scaffold for facile development of activatable heparanase probes bearing a variety of imaging moieties.
\end{abstract}

\section{Maintext}

Heparanase (HPSE) is an endo- $\beta$-glucuronidase that cleaves glycosidic bonds of heparan sulfate (HS) of heparan sulfate proteoglycans (HSPG) localized in the extracellular matrix and basement membrane. ${ }^{1}$ As HS plays critical roles in maintenance of integrity of the ECM and modulation of the activity of diverse, cytokines, chemokines and growth factors, the degradation of HS mediated by heparanase affected a variety of biological processes. ${ }^{2-4}$ Under normal physiological conditions, high-level heparanase can be detected in the placenta and blood-borne cells such as platelets, neutrophils, mast cells, and lymphocytes. ${ }^{3}$ Recently, the data revealed that heparanase is overexpressed in tumor tissues ${ }^{1,5-7}$ and the enzymatic activity is correlated with tumor metastasis, angiogenesis and post-surgical survival. ${ }^{8}$ Besides, 
the role of this enzyme is also associated with inflammatory disorders ${ }^{3,9}$ and autoimmune diabetes. ${ }^{10-11}$ Thus, it is unsurprising that heparanase has been regarded as a promising pharmacological target for treatment of multiple diseases.-4, 12-14

Recently, we reported the first structurally defined ultrasensitive fluorogenic probe HADP (1) for detecting heparanase activity with high selectivity and sensitivity, which we used in a high-throughput screen for novel heparanase inhibitors. ${ }^{15}$ In our initial probe design, use of the fluorogenic reporter 4methylumbelliferone (4-MU) did not facilitate turnover of the probe by HPSE, consistent with the exclusively endo-glycosidic selectivity of HPSE enzymatic activity. However, by incorporating two electron-withdrawing fluorine atoms on the methylumbelliferone reporter, ortho to the phenolic oxygen, our HADP probe successfully elicited exo-glycosidic activity from HPSE. ${ }^{15}$ Following this study, we developed a second fluorogenic probe for imaging heparanase activity in living cells (J.L., Z.W., and L.C., unpublished results), which also required two ortho-position fluorine atoms installed on the reporter, to elicit HPSE activity. Encouraged by these advances, we attempted to develop a near-infrared (NIR) probe for monitoring heparanase in vivo by extending the conjugation system of fluorophore, but the synthesis failed due to incompatibility of the NIR fluorophore with the chemical manipulation of the disaccharide recognition unit. Thus, we considered adding a self-immolative linker between the recognition unit and fluorescent reporter unit (Fig. 1). Typically, a self-immolative linker is based on a 4-hydroxybenzyl alcohol moiety to bridge the recognition unit and fluorophore. ${ }^{16-17}$ Once the recognition unit reacts with the corresponding analyte, the phenol of the middle linker is uncaged, which triggers simultaneous

A

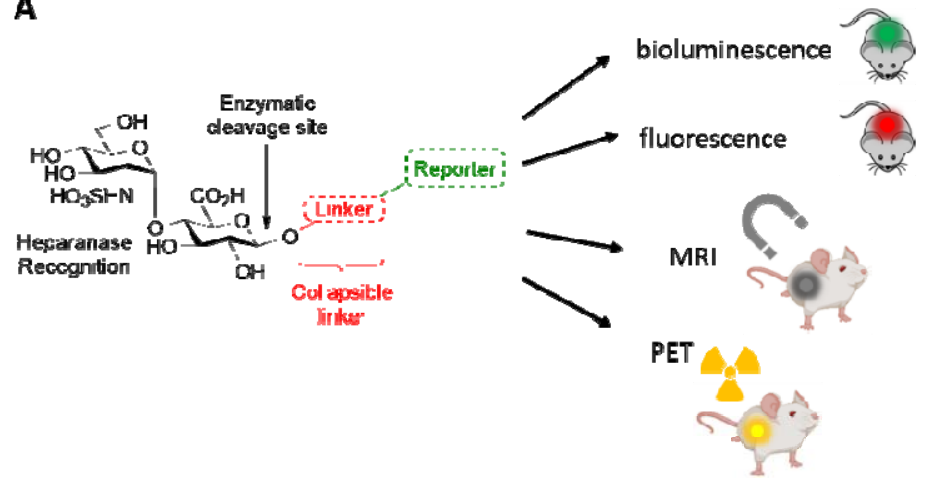

B

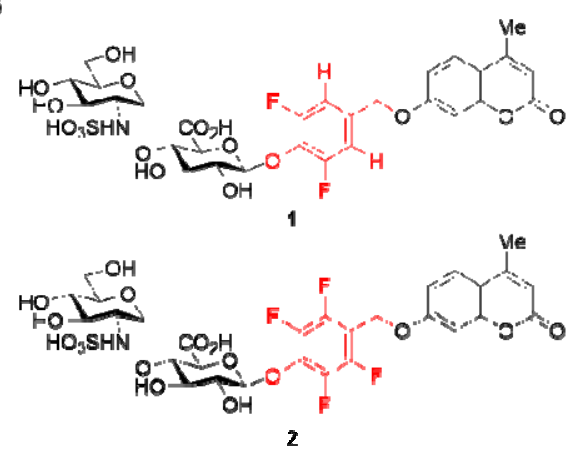

Fig. 1. (A) Design of HPSE probes bearing self-immolative linkers. This will serve as a scaffold for probes of many imaging modalities. (B) Probes $\mathbf{1}$ and $\mathbf{2}$ designed in this work, using differently tuned linkers.

elimination of a molecule of para quinone methide to release the latent reporter unit. This strategy has been extensively employed in the design of prodrugs, drug delivery, sensors, and molecular amplifiers. This self-immolative linker can efficiently reduce steric limitations and increase stability. Our previous 
work showed that $o, o$-difluorination of the fluorophore aglycone elicited heparanase activity while a non-fluorinated analog did not. This was due to the resultant weakening of the glycosidic bond and lowering of the transition state energy for enzymatic turnover by heparanase. ${ }^{15}$ Based on these results, we deigned two molecules bearing a 4-hydroxybenzyl alcohol linker substituted with either two or four fluorine atoms to facilitate enzymatic cleavage by heparanase. A simple coumarin as fluorescent reporter to examine whether the linker can be disassembled upon incubation with heparanase. Our results show that a tetrafluorobenzaldehyde (4-F) linker facilitates heparanase turnover, possibly providing a universal scaffold for small molecule heparanase probes.

We embarked on the synthesis for compounds 1 and $\mathbf{2}$ (Scheme 1) from disaccharide bromide 3 with 4-hydroxybenzaldehyde derivatives bearing 2 or 4 fluorine atoms by Koenigs-Knorr glycosylation to afford compounds $\mathbf{6}$ and $\mathbf{7}$, respectively. The aldehyde group was subsequently reduced to the primary
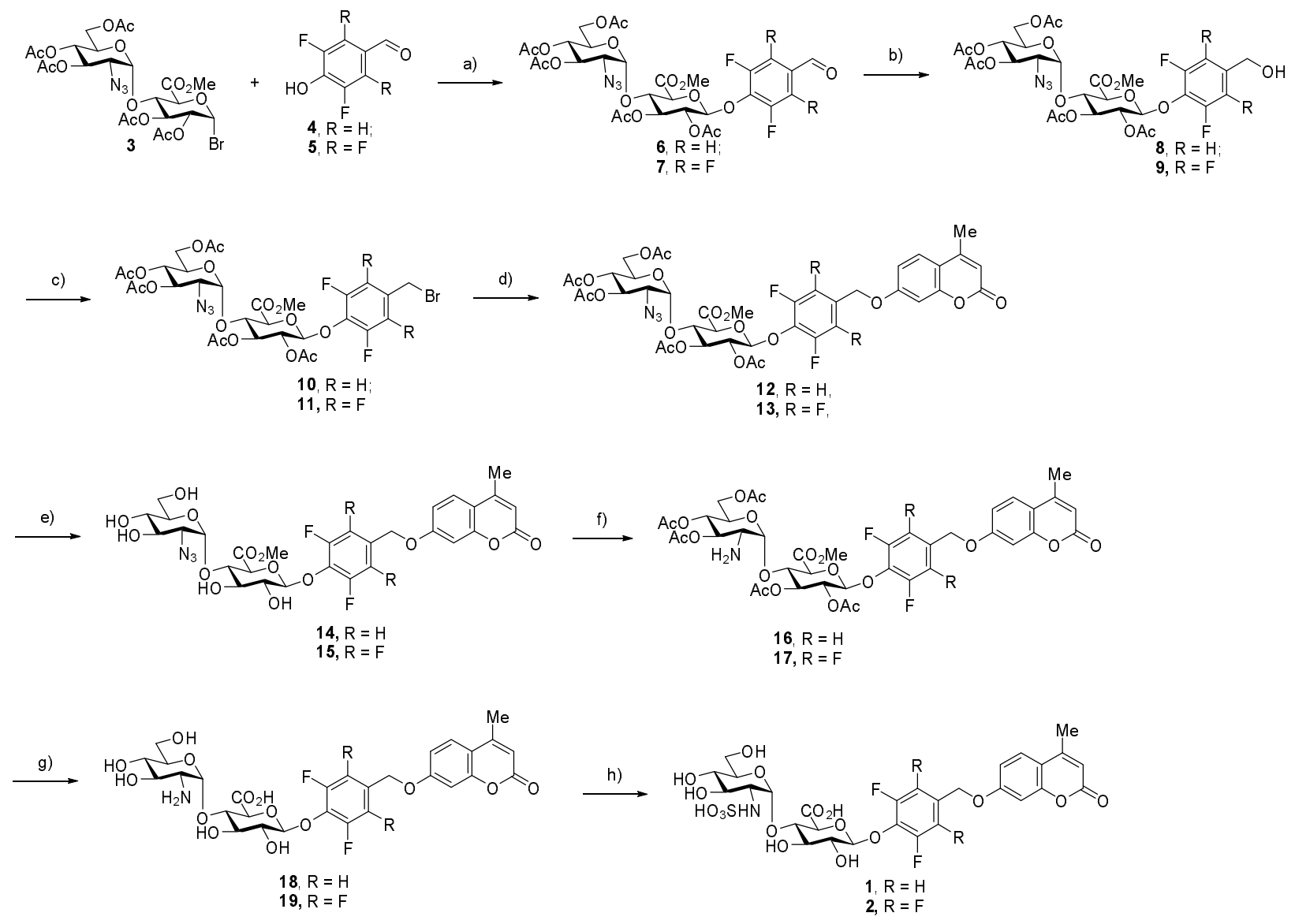

Scheme 1. Synthetic scheme for probes 1 and 2. Reagents and conditions: a) $\mathrm{Ag}_{2} \mathrm{O}, \mathrm{MeCN}$ (dry), r.t., $\mathrm{OVN}, \mathbf{7 7 \%}$ (6) or $54 \%$ (7); b) $\mathrm{NaBH}_{4}, \mathrm{DCM} / \mathrm{MeOH}(1 / 5)$, r.t., $20 \mathrm{~min}, 49 \%$ (8) or $64 \%$ (9); c) $\mathrm{PPh}_{3}, \mathrm{CBr}_{4}, \mathrm{DCM}$ (dry), r.t., 1 h, $46 \%$ (10) or 72\% (11); d) 4-MU, $\mathrm{K}_{2} \mathrm{CO}_{3}$, DMF, r.t., 16 h, 81\% (12) or 47\% (13); e) $\mathrm{NaOMe,} \mathrm{MeOH,} \mathrm{r.t.,} 2 \mathrm{~h}$, quant. (HPLC); f) Pd/C, $\mathrm{H}_{2}$, EtOAc, rt, 18 h, 75\% (HPLC); g) $\mathrm{NaOH}$ (pH 12), $\mathrm{H}_{2} \mathrm{O}, \mathrm{rt}, 4$ h, quant. (HPLC); h) Py. $\mathrm{SO}_{3}, \mathrm{NaOH}$ (pH 10), $\mathrm{H}_{2} \mathrm{O}, \mathrm{rt}, 6 \mathrm{~h}, 50 \%$ (HPLC). 
alcohol, followed by an Appel reaction to convert the alcohols to benzyl bromides $\mathbf{1 0}$ and $\mathbf{1 1}$. The alkyl bromides were substituted with 4-methylumbelliferone in the presence of potassium carbonate to incorporate the fluorescent reporter, establishing the skeleton of the desired molecules (compounds 12 and 13). Subsequent deprotection steps included Zemplén $O$-deacylation, catalytic hydrogenation of the azido group to the respective amine, and saponification of the glucuronic acid methyl ester. Finally, glucosamine $\mathbf{N}$-sulfation afforded compounds $\mathbf{1}$ and $\mathbf{2}$.

To confirm that the linkers work, we investigated the enzymatic response of compounds 1 and 2 to heparanase in $\mathrm{NaOAc}$ buffer ( $\mathrm{pH}$ 5.0) by measuring fluorescence turn-on from the (putatively) release methylumbelliferone reporter. For compound 1 with two fluorine atoms, negligible fluorescence was observed after incubation for 24 hours, indicating this linker could not be cleaved by heparanase.

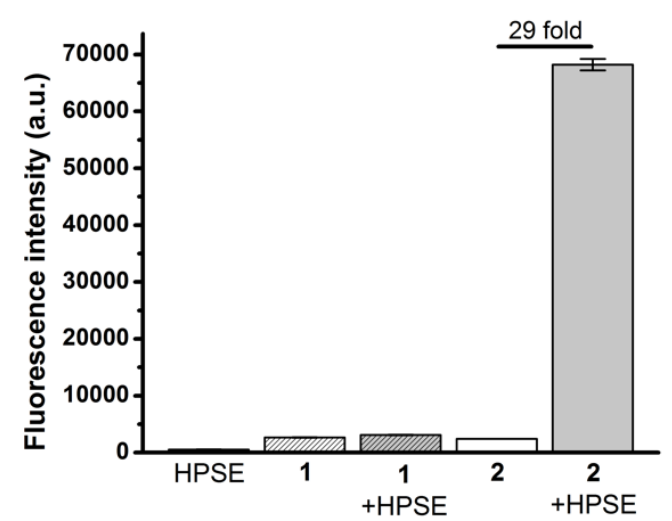

Fig. 2. Fluorescence emission of 1 and $\mathbf{2}$ upon incubation with HPSE after $24 \mathrm{~h}$. (B) Fluorescence of 2 after $24 \mathrm{~h}$ incubation, adjusted to $\mathrm{pH} 10$.
Furthermore, the reaction

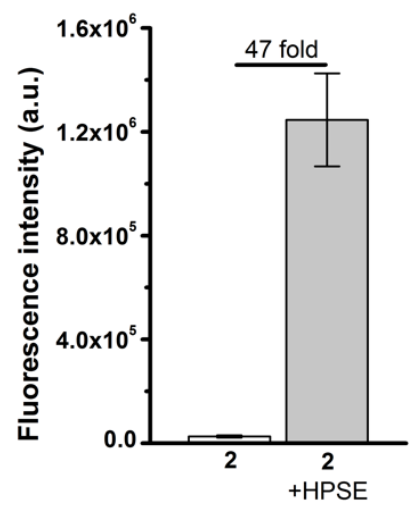

solution of this compound with heparanase was injected to HPLC. it shows that predominant peak remained, and the retention time was consistent with that of the compound 1. In contrast, compound 2 displayed a dramatic fluorescence enhancement after incubation for 3 hours in NaOAc buffer, at pH 5.0 (Fig. 2A). When the assay was terminated by increasing the $\mathrm{pH}$ to 10 , the turn-on ratio can be further boosted even with a diluted mixture (Fig. 2B). This cleavage is further confirmed by HPLC trace, giving a new peak in alignment with the corresponding free 4-methylumbelliferone in a complete conversion (Fig. 3). 


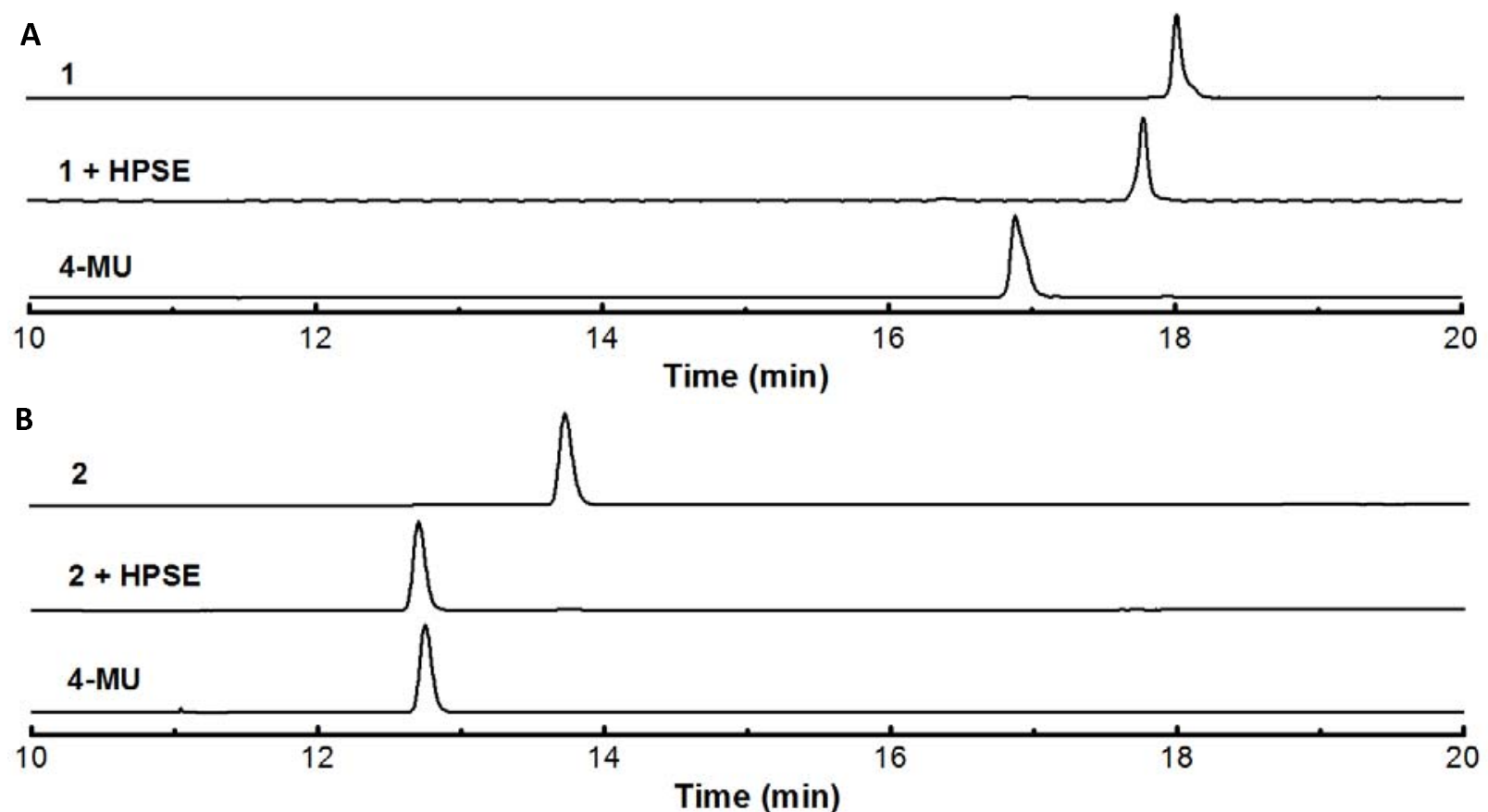

Fig. 3. HPLC trace of (A) 2-F or (B) 4-F with or without HPSE after $24 \mathrm{~h}$. Included is the free fluorophore, 4MU.

The successful response of $\mathbf{2}$ to HPSE suggests that the electronically tuned 4-F linker can facilitate turnover of probes using non-activated reporters, such as 7-MU. The 4-F scaffold thus opens up potential for the use of traditional reports in detecting HPSE activity, including commercially available fluorophores, chelates for magnetic or radioactive contrast, or smart imaging scaffolds, such as bioluminescence and in situ self-assembly moieties. The inclusion of 4-F avoids the requirement of functionalizing each of these reporters with EWGs such as the difluorination of our previously reported probe HADP, likely providing convenient and facile synthetic access to a library of novel HPSE imaging probes. We are currently synthesizing novel probes using the 4-F scaffold to expand this theory.

\section{Acknowledgements}

This work is supported by research grants to Prof. L. Cui from the National Institute of General Medical Sciences of National Institutes of Health (Maximizing Investigators' Research Award for Early Stage Investigators, R35GM124963), the Department of Defense (Congressionally Directed Medical Research Programs Career Development Award, W81XWH-17-1-0529), and to K.A. Schleyer from the University of Florida Health Cancer Center (UFHCC Predoctoral Award). We are grateful for the support from the University of Florida (UF) and the UF Health Cancer Center. NMR spectra were collected from the Department of Medicinal Chemistry, College of Pharmacy, University of Florida (UF). Mass spectrometry 
services were provided in part by the Mass Spectrometry Research and Education Center, Department of Chemistry, UF (supported by NIH S10 OD021758-01A1).

\section{Author contributions}

$\mathrm{JL}$ and $\mathrm{LC}$ designed the project. KAS, JL, and ZW synthesized the compounds. JL and KAS performed the fluorescence assays and HPLC characterization. KAS and LC wrote the paper. All authors reviewed the manuscript.

\section{Conflict of Interest}

The University of Florida has filed a patent on the work.

\section{Methods}

Experimental methods and chemical characterization are provided in the Supplementary Information.

\section{References}

1. Ilan, N.; Elkin, M.; Vlodavsky, I., Regulation, function and clinical significance of heparanase in cancer metastasis and angiogenesis. Int J Biochem Cell Biol 2006, 38 (12), 2018-39.

2. Vlodavsky, I.; Friedmann, Y.; Elkin, M.; Aingorn, H.; Atzmon, R.; Ishai-Michaeli, R.; Bitan, M.; Pappo, O.; Peretz, T.; Michal, I.; Spector, L.; Pecker, I., Mammalian heparanase: gene cloning, expression and function in tumor progression and metastasis. Nat Med 1999, 5 (7), 793-802.

3. Rivara, S.; Milazzo, F. M.; Giannini, G., Heparanase: a rainbow pharmacological target associated to multiple pathologies including rare diseases. Future Med Chem 2016, 8 (6), 647-80.

4. Rabelink, T. J.; van den Berg, B. M.; Garsen, M.; Wang, G.; Elkin, M.; van der Vlag, J., Heparanase: roles in cell survival, extracellular matrix remodelling and the development of kidney disease. Nat Rev Nephrol 2017, 13 (4), 201-212.

5. Fux, L.; Ilan, N.; Sanderson, R. D.; Vlodavsky, I., Heparanase: busy at the cell surface. Trends Biochem Sci 2009, 34 (10), 511-9.

6. Vreys, V.; David, G., Mammalian heparanase: what is the message? J Cell Mol Med 2007, 11 (3), 42752.

7. Vlodavsky, I.; Goldshmidt, O.; Zcharia, E.; Atzmon, R.; Rangini-Guatta, Z.; Elkin, M.; Peretz, T.; Friedmann, Y., Mammalian heparanase: involvement in cancer metastasis, angiogenesis and normal development. Semin Cancer Biol 2002, 12 (2), 121-9.

8. Cohen, E.; Doweck, I.; Naroditsky, I.; Ben-Izhak, O.; Kremer, R.; Best, L. A.; Vlodavsky, I.; Ilan, N., Heparanase is overexpressed in lung cancer and correlates inversely with patient survival. Cancer 2008, 113 (5), 1004-11.

9. Li, J. P.; Vlodavsky, I., Heparin, heparan sulfate and heparanase in inflammatory reactions. Thromb Haemost 2009, 102 (5), 823-8.

10. Simeonovic, C. J.; Ziolkowski, A. F.; Wu, Z.; Choong, F. J.; Freeman, C.; Parish, C. R., Heparanase and autoimmune diabetes. Front Immunol 2013, 4, 471.

11. Ziolkowski, A. F.; Popp, S. K.; Freeman, C.; Parish, C. R.; Simeonovic, C. J., Heparan sulfate and heparanase play key roles in mouse beta cell survival and autoimmune diabetes. $J$ Clin Invest 2012, 122 (1), 132-41. 
12. McKenzie, E. A., Heparanase: a target for drug discovery in cancer and inflammation. Br J Pharmacol 2007, 151 (1), 1-14.

13. Mohan, C. D.; Hari, S.; Preetham, H. D.; Rangappa, S.; Barash, U.; Ilan, N.; Nayak, S. C.; Gupta, V. K.; Basappa; Vlodavsky, I.; Rangappa, K. S., Targeting Heparanase in Cancer: Inhibition by Synthetic, Chemically Modified, and Natural Compounds. iScience 2019, 15, 360-390.

14. Masola, V.; Zaza, G.; Gambaro, G.; Franchi, M.; Onisto, M., Role of heparanase in tumor progression: Molecular aspects and therapeutic options. Semin Cancer Biol 2020, 62, 86-98.

15. Liu, J.; Schleyer, K. A.; Bryan, T. L.; Xie, C.; Seabra, G.; Xu, Y.; Kafle, A.; Cui, C.; Wang, Y.; Yin, K.; Fetrow, B.; Henderson, P. K. P.; Fatland, P. Z.; Liu, J.; Li, C.; Guo, H.; Cui, L., Ultrasensitive small molecule fluorogenic probe for human heparanase. Chemical Science 2021, 12 (1), 239-246.

16. Yan, J.; Lee, S.; Zhang, A.; Yoon, J., Self-immolative colorimetric, fluorescent and chemiluminescent chemosensors. Chem. Soc. Rev. 2018, 47 (18), 6900-6916.

17. Alouane, A.; Labruere, R.; Le Saux, T.; Schmidt, F.; Jullien, L., Self-immolative spacers: kinetic aspects, structure-property relationships, and applications. Angew. Chem. Int. Ed. 2015, 54 (26), 7492-509. 